EPJ Web of Conferences 95,01004 (2015)

DOI: $10.1051 /$ epjconf/ 20159501004

C) Owned by the authors, published by EDP Sciences, 2015

\title{
Parton/hadron dynamics in heavy-ion collisions at FAIR energies
}

\author{
W. Cassing ${ }^{1, a}$, V. P. Konchakovski ${ }^{1}$, A. Palmese ${ }^{1}$, V. D. Toneev², and E. L. Bratkovskaya ${ }^{3}$ \\ ${ }^{1}$ Institut für Theoretische Physik, University of Giessen, Germany \\ ${ }^{2}$ Joint Institute for Nuclear Research, 141980 Dubna, Russia \\ ${ }^{3}$ Institut für Theoretische Physik, Johann Wolfgang Goethe University Frankfurt/M, Germany
}

\begin{abstract}
Recent STAR data for the directed flow of protons, antiprotons and charged pions obtained within the beam energy scan program are analyzed within the PartonHadron-String-Dynamics (PHSD/HSD) transport models. Both versions of the kinetic approach are used to clarify the role of partonic degrees of freedom. The PHSD results, simulating a partonic phase and its coexistence with a hadronic one, are roughly consistent with the STAR data. Generally, the semi-qualitative agreement between the measured data and model results supports the idea of a crossover type of quark-hadron transition which softens the nuclear EoS but shows no indication of a first-order phase transition. Furthermore, the directed flow of kaons and antikaons is evaluated in the PHSD/HSD approachesfrom $\sqrt{s_{N N}} \approx 3-200 \mathrm{GeV}$ which shows a high sensitivity to hadronic potentials in the FAIR/NICA energy regime $\sqrt{s_{N N}} \leq 8 \mathrm{GeV}$.
\end{abstract}

\section{Introduction}

The study of the particle azimuthal angular distribution in momentum space with respect to the reaction plane is an important tool to probe the hot, dense matter created in heavy-ion collisions [1, 2]. The directed flow refers to a collective sidewards deflection of particles and is characterized by the first-order harmonic $v_{1}$ of the Fourier expansion of the particle azimuthal angular distribution with respect to the reaction plane [3]. The second harmonic coefficient $v_{2}$, called elliptic flow, and the triangular flow $v_{3}$ have been extensively studied both theoretically and experimentally in the last years by about five orders of magnitude in the collision energy $\sqrt{s_{N N}}$ [4]. In contrast, apart from first measurements in the early nineties and till recent times, the directed flow was studied mainly theoretically [5-7] although some experimental information from the Schwerionen-Synchrotron (SIS) to Super-Proton-Synchrotron (SPS) energies is available [8].

It is generally assumed that the directed flow is generated during the nuclear passage time $[9,10]$. The directed transverse flow therefore probes the onset of bulk collective dynamics during thermalization, thus providing valuable information on the pre-equilibrium stage [11-14]. In earlier times (at moderate beam energies) the first flow harmonic defined as

$$
v_{1}(y)=\left\langle\cos \left(\phi-\phi_{R P}\right)\right\rangle=\left\langle v_{x} / \sqrt{v_{x}^{2}+v_{y}^{2}}\right\rangle
$$

ae-mail: Wolfgang.Cassing@theo.physik.uni-giessen.de

This is an Open Access article distributed under the terms of the Creative Commons Attribution License 4.0, which permits unrestricted use, distribution, and reproduction in any medium, provided the original work is properly cited. 
with respect to the reaction plane $\phi_{R P}$ was characterized differently: i.e. by the mean transverse momentum per particle projected on the reaction $(x-z)$ plane $\left\langle p_{x}(y) / N\right\rangle$ in the center-of-mass system which differs from the $v_{1}$ harmonic component. Unfortunately, it is not possible to convert or directly compare $v_{1}$ data to the earlier $p_{x} / N$ analysis. The NA49 collaboration [15] has measured the flow coefficient $\mathrm{v}_{1}$ for pions and protons at SPS energies and a negative $v_{1}(y)$ slope was observed by the standard event plane method for pions. Often, just the slope of $v_{1}(y)$ at midrapidity has been used to quantify the strength of the directed flow.

At Alternating-Gradient-Synchrontron (AGS) energies $E_{\text {lab }} \lesssim 11.5 \mathrm{~A} \cdot \mathrm{GeV}$, the $v_{1}$ dependence has a characteristic $\mathrm{S}$-shape attributed to the standard $\left\langle p_{x}(y) / N\right\rangle$ distribution. The projected average momentum $<p_{x}(y)>$ grows linearly with rising rapidity $y$ between the target and projectile fragmentation regions. Conventionally, this type of flow- with positive derivative $d v_{1} / d y$ - is called normal flow, in contrast to the antiflow for which $d v_{1} / d y<0$ [10,15-17]. At these moderate energies the slope of $v_{1}(y)$ at midrapidity $(F)$ is observed to be positive for protons and significantly smaller in magnitude and negative for pions $[15,16,18]$. The smooth fall-off of this function with beam energy is reasonably reproduced by the available hadronic kinetic models (see the comparison in Ref. [19]).

The shape of the rapidity dependence $v_{1}(y)$ with bombarding energy is of special interest because the directed flow at midrapidity may be modified by the collective expansion and reveal a signature of a phase transition from normal nuclear matter to a quark-gluon plasma (QGP). This is commonly studied by measuring the central rapidity region that reflects important features of the system evolution from its initial state. The predicted $v_{1}(y)$ flow coefficient is small close to midrapidity with almost no dependence on pseudorapidity. However, as first demonstrated in Refs. [20, 21], the 3D hydrodynamic expansion - with an equation of state (EoS) including a possible phase transition - exhibits some irregularity in the evolution of the system. When including a first order phase transition this leads to a local minimum in the proton excitation function of the transverse directed flow at $E_{l a b} \approx 8 \mathrm{~A} \cdot \mathrm{GeV}$. Such a first order transition leads to a softening of the equation of state and consequently to a time-delayed expansion. The existence of this 'softest point' of the EoS at a minimum of the energy density $\varepsilon_{S P}$ leads to a long lifetime of the mixed phase and consequently in a prolonged expansion of matter [22]. Presently, the critical energy density (or latent heat for a first order transition at finite quark chemical potential) is not well known and estimates vary from $0.5 \mathrm{GeV} / \mathrm{fm}^{3}$ to $1.5 \mathrm{GeV} / \mathrm{fm}^{3}$ [22-25]. A softest point at $\varepsilon_{S P} \sim 1.5 \mathrm{GeV} / \mathrm{fm}^{3}$ should give a minimum in the directed flow excitation function at $E_{\text {lab }} \sim$ $30 \mathrm{GeV} \mathrm{A} \cdot \mathrm{GeV}[22,23]$. In case of ideal hydrodynamics the directed proton flow $p_{x}$ shows even a negative $v_{1}$ (' $v_{1}$ collapse') between $E_{l a b}=8$ and $20 \mathrm{~A} \cdot \mathrm{GeV}$ [26] and with rising energy increases back to a positive flow. The ideal hydro calculations suggest that this 'softest point collapse' is at $E_{l a b} \sim 8 \mathrm{~A} \cdot \mathrm{GeV}$ but this was not confirmed by available AGS data [26]. A linear extrapolation of the AGS data indicates that a collapse of the directed proton flow might be at $E_{\text {lab }} \approx 30 \mathrm{~A} \cdot \mathrm{GeV}$. However, this minimum in the given energy range is not supported in the two-fluid model with a phase transition [19].

The interest in the directed flow $v_{1}(y)$ has recently been enhanced considerably due to new STAR data obtained in the framework of the beam energy scan (BES) program [27]. The directed flow of identified hadrons - protons, antiprotons, positive and negative pions - has been measured with high precision for semi-central $\mathrm{Au}+\mathrm{Au}$ collisions in the energy range $\sqrt{s_{N N}}=(7.7-39) \mathrm{GeV}$. These data provide a promising basis for studying direct-flow issues as discussed above and have been addressed already by the Frankfurt group [28] limiting themselves to the energy $\sqrt{s_{N N}}<20 \mathrm{GeV}$ where hadronic processes are expected to be dominant. However, the authors of Ref. [28] did not succeed to describe the data and to obtain conclusive results which led to the notion of the 'directed flow puzzle'. Our study aims to analyze these STAR results in the whole available energy range including in particular antiproton data [29]. 
We start with a short reminder of the PHSD approach and its hadronic version HSD (without partonic degrees of freedom) and then analyse the BES data in terms of both transport models in order to explore where effects from partonic degrees of freedom show up. Furthermore, we compare also with predictions of other kinetic models in Sec. II. In Sec. III we provide predictions for kaon and antikaon directed flows for $\mathrm{Au}+\mathrm{Au}$ collisions from $\sqrt{s_{N N}} \approx 3-200 \mathrm{GeV}$ and investigate in particular the sensitivity to hadronic potentials. Our findings are summarized in Sec. IV.

\section{Directed flow in microscopic approaches}

\subsection{Reminder of PHSD}

The PHSD model is a covariant dynamical approach for strongly interacting systems formulated on the basis of Kadanoff-Baym equations [30, 31] or off-shell transport equations in phase-space representation, respectively. In the Kadanoff-Baym theory the field quanta are described in terms of dressed propagators with complex selfenergies. Whereas the real part of the selfenergies can be related to mean-field potentials of Lorentz scalar, vector or tensor type, the imaginary parts provide information about the lifetime and/or reaction rates of time-like particles [32]. Once the proper complex selfenergies of the degrees of freedom are known, the time evolution of the system is fully governed by off-shell transport equations for quarks and hadrons (as described in Refs. [30, 32]). The PHSD model includes the creation of massive quarks via hadronic string decay - above the critical energy density $\sim 0.5 \mathrm{GeV} / \mathrm{fm}^{3}$ - and quark fusion forming a hadron in the hadronization process. With some caution, the latter process can be considered as a simulation of a crossover transition since the underlying EoS in PHSD is a crossover [32]. At energy densities close to the critical energy density the PHSD describes a coexistence of the quark-hadron mixture. This approach allows for a simple and transparent interpretation of lattice QCD results for thermodynamic quantities as well as correlators and leads to effective strongly interacting partonic quasiparticles with broad spectral functions. For a review on off-shell transport theory we refer the reader to Ref. [32]; PHSD model results and their comparison with experimental observables for heavy-ion collisions from the lower super-proton-synchrotron (SPS) to RHIC energies can be found in Refs. [32-35]. In the hadronic phase, i.e. for energies densities below the critical energy density, the PHSD approach is identical to the Hadron-String-Dynamics (HSD) model [36-38].

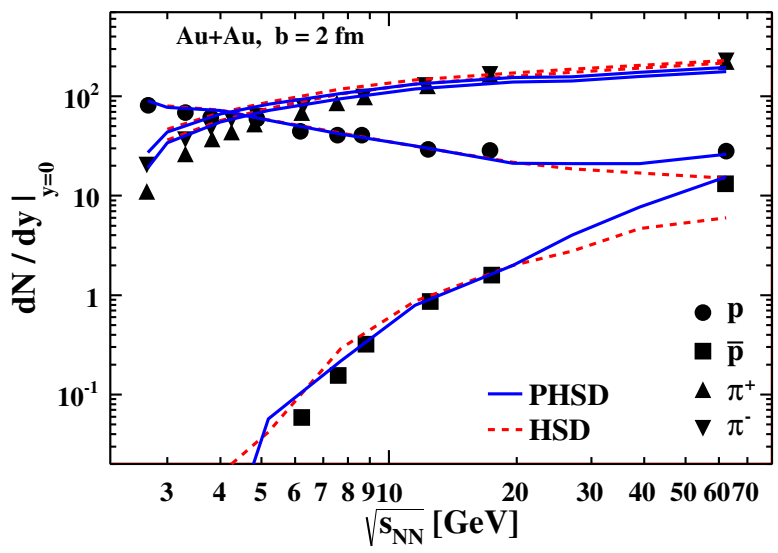

Figure 1. Particle abundance at mid-rapidity calculated for central collisions $b=2 \mathrm{fm}$ in the HSD (dashed lines) and PHSD (solid lines) models. The experimental data are from a compilation of Ref. [40] complemented by recent data from the STAR collaboration [41] and the latest update of the compilation of NA49 results $[42,43]$.

The HSD approach formally can be written as a coupled set of transport equations for the phasespace distributions $f_{h}(x, p)$ of hadron $h$, which includes the real part of the scalar and vector hadron 
self-energies. The hadron quasi-particle properties here are defined via the mass-shell constraint with effective masses and momenta. In the HSD transport calculations we include nucleons, $\Delta$ 's, $N^{*}(1440), N^{\star}(1535), \Lambda, \Sigma$ and $\Sigma^{\star}$ hyperons, $\Xi$ 's and $\Omega$ 's as well as their antiparticles. High energy inelastic hadron-hadron collisions are described by the FRITIOF model [44], where two incoming hadrons emerge the reaction as two excited color singlet states, i.e. 'strings'. The excitation functions for various dynamical quantities as well as experimental observables from SIS to RHIC energies within the HSD transport approach can be found in Refs. [37-39].

Fig. 1 illustrates how the hadron multiplicity $d N / d y(y=0)$ at midrapidity is reproduced within the PHSD (solid lines) and HSD (dashed lines) kinetic approaches. We point out that the antiproton abundance is a crucial issue. In the AGS-SPS low energy range $(\leq 20 \mathrm{GeV})$ both models agree quite reasonably with experiment, including the antiproton yield. The enhancement of the proton and antiproton yield at $\sqrt{s_{N N}}=62 \mathrm{GeV}$ in PHSD relative to HSD can be traced back to a larger baryon/antibaryon fraction in the hadronization process. At lower energies this agreement is reached by taking into account the $p \bar{p}$ annihilation to three mesons (e.g. $\pi, \rho, \omega)$ as well as the inverse channels employing detailed balance as worked out in Ref. [45]. These inverse channels are quite important; in particular, at the top SPS energy this inverse reaction practically compensates the loss of antiprotons due to their annihilation [45]. At lower SPS and AGS energies the annihilation is dominant due to the lower meson abundancies, however, the backward channels reduce the net annihilation rate. We mention that the multiple-meson recombination channels are not incorporated in the standard UrQMD transport model [46]. The proton multiplicities are reproduced rather well in the PHSD/HSD approaches but the multiplicity of charged pions is slightly overestimated for $\sqrt{s_{N N}} \leq 10 \mathrm{GeV}$. This discrepancy is observed also in other transport models [47, 48].

\subsection{Directed flow from microscopic transport models}

The whole set of directed flow excitation functions for protons, antiprotons and charged pions from the PHSD/HSD models is presented in Fig. 2 (1.h.s.) in comparison to the measured data [27]. The initial states in the PHSD/HSD are simulated on an event-by-event basis taking into account fluctuations in the position of the initially colliding nucleons and fluctuations in the reaction plane. This procedure is identical to that in the study of the elliptic flow in Ref. [33]. The average impact parameter for the selected events is $b=7 \mathrm{fm}$. In the simulations the experimental acceptance $0.2 \geq p_{T} \geq 2 \mathrm{GeV} / \mathrm{c}$ is taken into account for all hadrons [27]. Note that the PHSD/HSD calculations in Fig. 2 have been performed without incorporating any hadronic mean fields for $\sqrt{s_{N N}} \geq 7.7 \mathrm{GeV}$.

At first glance, both models - in particularly the PHSD - correctly reproduce the general trends in the differential $v_{1}(y)$ with bombarding energy: the $v_{1}(y)$ slope for protons is positive at low energies $\left(\sqrt{s_{N N}} \leq 20 \mathrm{GeV}\right)$ and approaches zero with increasing energy while antiprotons and pions have negative slopes, respectively, in the whole energy range. In more detail: for protons the directed flow distributions are in a reasonable agreement with the STAR measurements in the whole range of the collision energies considered (except for $\sqrt{s_{N N}}=11.5 \mathrm{GeV}$ ). However, $v_{1}(y)$ for antiprotons agrees with the data only for the highest energies where baryon/antibaryon pairs are dominantly produced by hadronization. This becomes evident from a comparison to the HSD results with $v_{1}(y) \approx 0$. The shape of the $v_{1}(y)$ distribution for antiprotons starts progressively to differ from the measured data if we proceed from $\sqrt{s_{N N}}=11.5$ down to $7.7 \mathrm{GeV}$. In the lower energy range the HSD and PHSD results get very close which indicates the dominance of hadronic reaction channels (absorption and recreation). The direct flow distributions for negative and positive pions are close to each other and also begin to disagree with experiment in the same range of low collision energies as for antiprotons (see Fig. 2 (l.h.s.)). Again the PHSD results are very close to the experimental measurements at higher energies while the HSD results deviate more sizeably thus stressing the role of partonic degrees of freedom in 

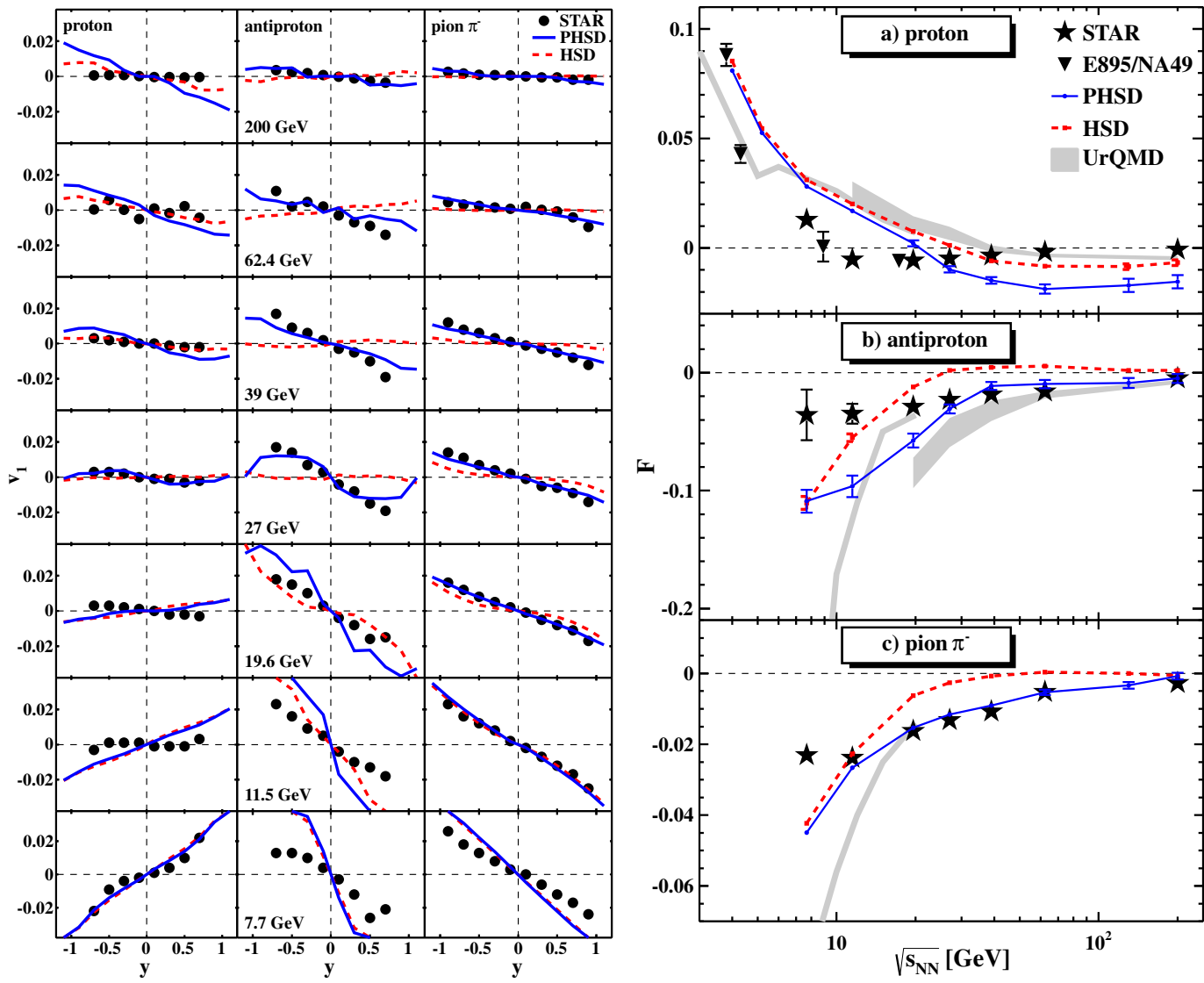

Figure 2. (1.h.s.) The directed flow $v_{1}(y)$ for protons, antipprotons as well as negative pions from $\mathrm{Au}+\mathrm{Au}$ collisions at different collision energies from $\sqrt{s_{N N}}=7.7$ to $39 \mathrm{GeV}$ from HSD (dashed lines) and PHSD (solid lines). Experimental data are from the STAR collaboration [27]. (r.h.s.) The beam energy dependence of the directed flow slope $F$ at midrapidity for protons, antiproton and charged pions from semicentral $\mathrm{Au}+\mathrm{Au}$ collisions. The shaded band corresponds to the UrQMD results as cited in [27]. The experimental data are from the STAR collaboration [27] along with results of prior experiments using comparable cuts [15, 50]. The PHSD/HSD calculations have been performed without incorporating any hadronic mean fields.

the entire collision dynamics. The clear overestimation of the $\bar{p}$ and $\pi^{-}$slopes at $\sqrt{s_{N N}}=7.7 \mathrm{GeV}$ demonstrates that the heavy-ion dynamics is not yet fully understood within the string/hadron picture at the lower energies without incorporating any hadronic mean fields.

The characteristic slope of the $v_{1}(y)$ distributions at midrapidity, $\left.\frac{d v_{1}}{d y}\right|_{y=0}=F$, is presented in Fig. 2 (r.h.s.) for all cases considered. In a first approximation the $v_{1}$ flow in the center-of-mass system may be well fitted by a linear function $v_{1}(y)=F y$ within the rapidity interval $-0.5<y<0.5$. A cubic equation is also used,

$$
v_{1}(y)=F y+C y^{3},
$$

to obtain an estimate of the uncertainty in extracting the coefficient $F$. The error bars in Fig. 2 (r.h.s.) just stem from the different fitting procedures. Note that the energy axis in Fig. 2 (r.h.s.) is extended 
by adding experimental results for $\sqrt{s_{N N}}=62$ and $200 \mathrm{GeV}$ [27]. This representation is more delicate as compared to $v_{1}(y)$ in Fig. 2 (1.h.s.). For protons there is a qualitative agreement of the HSD/PHSD results with the experiment measurements: the slope $F>0$ at low energies, however, exceeding the experimental values by up a factor of about two; the slope crosses the line $F=0$ at $\sqrt{s_{N N}} \sim 20$ $\mathrm{GeV}$, which is twice larger than the experimental crossing point, and then stays negative and almost constant with further energy increase. However, the absolute values of the calculated proton slopes in this high energy range are on the level of -(0.010-0.015), while the measured ones are about 0.005 . The standard UrQMD model results, as cited in the experimental paper [27] and in the more recent theoretical work [28], are displayed in Fig. 2 (r.h.s.) by the wide and narrow shaded areas, respectively. These results for protons are close to those from the HSD and essentially overestimate the slope for energies below $\sim 30 \mathrm{GeV}$ but at higher energy become negative and relatively close to the experiment. The predictions for the pure hadronic version of the transport model HSD (dotted lines in Fig. 2 (r.h.s.) slightly differ from the PHSD results which overpredict the negative proton slope at higher RHIC energies.

For the antiproton slopes we again observe an almost quantitative agreement with the BES experiment [27]: with increasing collision energy the HSD and PHSD slopes grow and then flatten above 20-30 GeV. The HSD results saturate at $v_{1}(0)=0$, while the PHSD predictions stay negative and in good agreement with experiment (see Fig. 2 (r.h.s.). It is noteworthy to point out that these PHSD predictions strongly differ from the UrQMD results which no longer describe the data for $\sqrt{s_{N N}} \leq 20$ $\mathrm{GeV}$ but are in agreement with the measurements for higher energies. This disagreement might be attributed to a neglect of the inverse processes for antiproton annihilation [45] in UrQMD as described above.
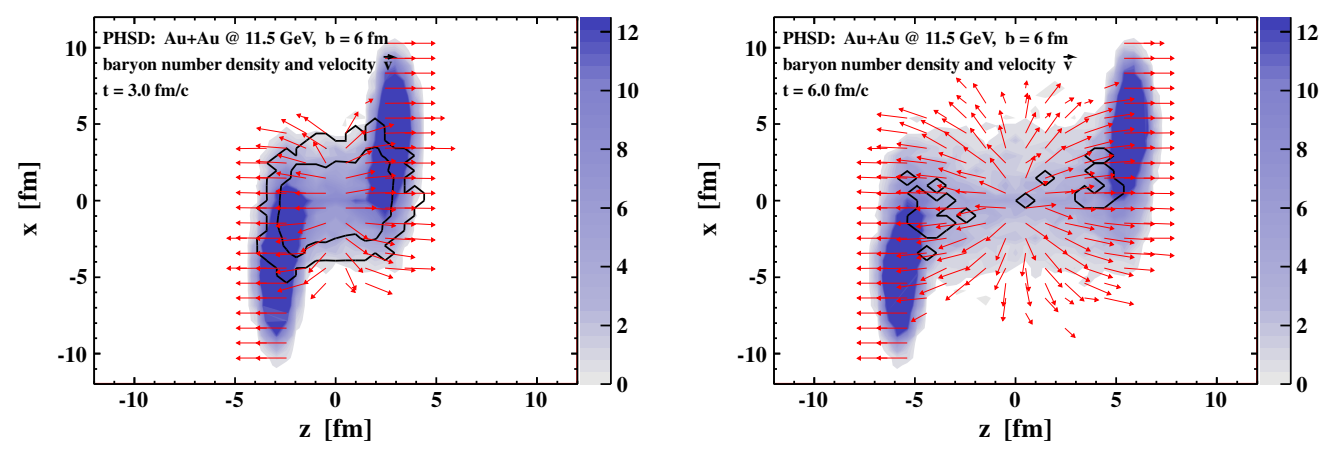

Figure 3. Snapshots of the baryon energy density distribution in the PHSD model at the time $t=3 \mathrm{fm} / \mathrm{c}$ and 6 $\mathrm{fm} / \mathrm{c}$ for $\mathrm{Au}+\mathrm{Au}$ collisions and $\sqrt{s_{N N}}=11.5 \mathrm{GeV}$. The energy density scale is given on the right side in $\mathrm{GeV} / \mathrm{fm}^{3}$. The solid curves display parton density levels for 0.6 and 0.01 partons $/ \mathrm{fm}^{3}$. The arrows show the local velocity of baryonic matter (in relative units).

The differences between the calculations and experimental data become apparent for the charged pion slopes at $\sqrt{s_{N N}} \leq 11 \mathrm{GeV}$ : the negative minimum of the charged pion slope is deeper than the measured one. The HSD and PHSD results practically coincide at low energy (due to a minor impact of partonic degrees of freedom) but dramatically differ from those of the UrQMD model for $\sqrt{s_{N N}} \leq 20$ $\mathrm{GeV}$ (see Fig.2 (r.h.s.). This difference might be attributed again to a neglect of the inverse processes for antiproton annihilation in UrQMD. 
The appearance of negative $v_{1}$-slopes can be explained by the evolution of the tilted ellipsoid-like shape of the participant zone. Snapshots of the velocity profile are shown in Fig. 3 for times $t=3 \mathrm{fm} / \mathrm{c}$ and $6 \mathrm{fm} / \mathrm{c}$ for semi-peripheral $\mathrm{Au}+\mathrm{Au}(11.5 \mathrm{GeV})$ collisions in the background of baryon density distributions where also parton blobs can be identified. Indeed, among the scattered particles there are many which move perpendicularly to the stretched matter (antiflow) and their multiplicity increases with time.
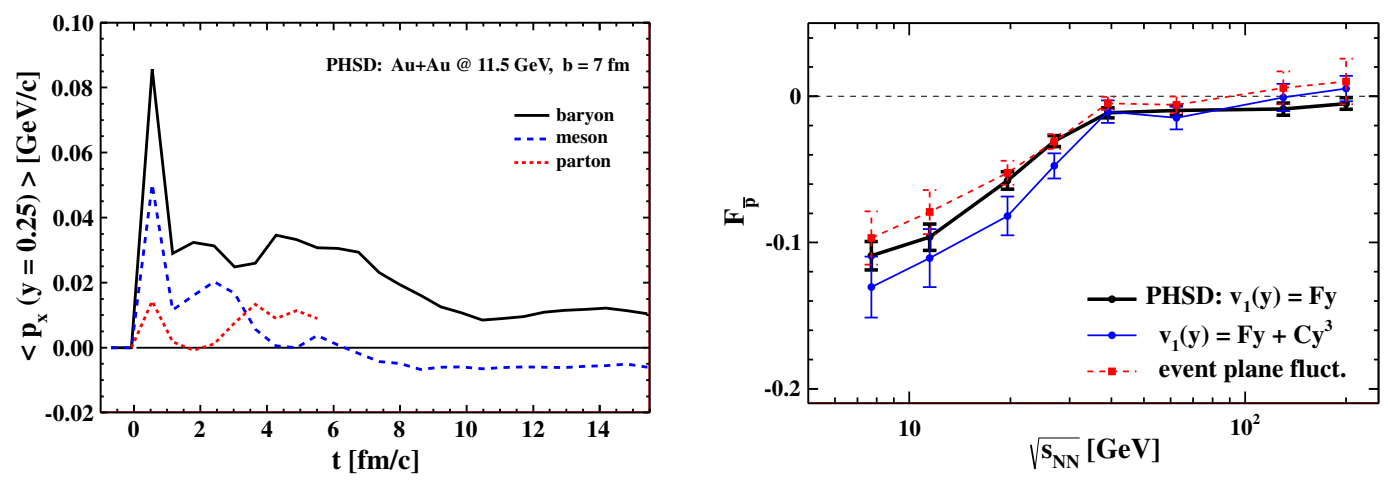

Figure 4. (1.h.s.) Evolution of the average momentum projection on the reaction plane for protons, pions and quarks at the shifted rapidity $y=0.25 \pm 0.05$. The results are given for $8.710^{4} \mathrm{PHSD}$ events of Au+Au collisions at $\sqrt{s_{N N}}=11.5 \mathrm{GeV}$. (r.h.s.) Excitation function of the antiproton slope $F_{\bar{p}}$ calculated in the PHSD model with (dotted line) and without (solid line) including fluctuations of the reaction plane. The dotted line corresponds to a use of the cubic equation (2) for the slope calculation.

However, this component is weak and it is not clear whether these snapshots will result in observable effects for the final slope. The solution of this question is shown in Fig. 4 (1.h.s.). Here it is clearly seen that the directed flow is formed at an early stage of the nuclear interaction. Then, the $v_{1}$ flow decreases for protons and pions reaching positive and negative slopes, respectively, in accordance with the results in Fig. 2. Thus, in agreement with the STAR experimental data, in the considered energy range the PHSD model predicts for protons a smooth $F\left(\sqrt{s_{N N}}\right)$ function which is flattening at $\sqrt{s_{N N}} \geq 10 \mathrm{GeV}$ and reveals no signatures of a possible first-order phase transition as expected in Refs. [20-26]. For antiprotons the slope at midrapidity manifests a wide but shallow negative minimum for $\sqrt{s_{N N}} \approx 30 \mathrm{GeV}$ while the measured slope is a monotonically increasing function. It is noteworthy that the new STAR data are consistent with the PHSD results which include a crossover transition by default due to a matching of the EoS to lattice QCD results. We note in passing that fluctuations of the reaction plane give only a small effect on the directed flow of hadrons which is most pronounced for antiprotons (cf. Fig. 4 (r.h.s.)).

\section{Directed flow of kaons and antikaons}

Strange hadrons and in particulalar kaons and antikaons provide additional information on the reaction dynamics. In relativistic mean-field models the dispersion relation for kaons and antikaons in the nuclear medium can be written as [53-56]

$$
\omega_{K^{ \pm}}^{2}\left(\rho_{N}, \mathbf{p}\right)= \pm \frac{3}{4} \frac{\omega}{f_{K}^{2}} \rho_{N}+m_{K}^{2}+\mathbf{p}^{2}-\frac{\Sigma_{K N}}{f_{K}^{2}} \rho_{s} .
$$


In Eq. (3) $\Sigma_{K N}$ is the kaon-nucleon sigma term $(\approx 400 \mathrm{MeV}), m_{K}$ denotes the bare kaon mass, $f_{K} \approx 100$ $\mathrm{MeV}$ is the kaon decay constant, while $\rho_{s}$ and $\rho_{N}$ stand for the scalar and vector nucleon densities, respectively. This leads to repulsive kaon mean fields $U_{K}>0$ and attractive mean fields for the antikaons $U_{\bar{K}}<0$ at finite baryon density $[53,54,56]$.

\section{1 $K^{ \pm}$potentials}

The microscopic calculation of kaon and antikaon potentials (or mean fields) is more involved and can e.g. be worked out within G-matrix theory $[57,58]$. These calculations show that the potentials also explicitly depend on the $K^{ \pm}$momentum $p$ with respect to the local rest frame of the system. Such a momentum-dependence can be incorporated by introducing momentum dependent formfactors in the scalar and vector potentials and fitting the parameters to the results from G-matrix theory up to twice nuclear matter density. Any extrapolation to higher densities, however, should be taken with great care since no robust information is available so far. In order to shed some light on the possible effects of $K^{ \pm}$potentials on their directed flow - especially at FAIR/NICA energies - we incorporated the momentum-dependent potentials for kaons and antikaons as displayed in Fig. 5 as a function of the momentum $p$ for nuclear densities $\rho_{N}$ from $0.1-1.0 \mathrm{fm}^{-3}$ in steps of $0.1 \mathrm{fm}^{-3}$. These potentials, defined by

$$
U_{K}\left(\mathbf{p}, \rho_{N}\right)=\omega_{K}\left(\rho_{N}, \mathbf{p}\right)-\sqrt{\mathbf{p}^{2}+m_{K}^{2}},
$$

typically increase/decrease with momentum and asymptotically tend to zero again. Note that a density of $1.0 \mathrm{fm}^{-3}$ roughly corresponds to 6 times nuclear matter density where the system no longer should consist of hadronic degrees of freedom. Accordingly these potentials are dominantly probed only up to 3 times nuclear matter density in actual PHSD calculations since the partonic degrees of freedom take over at higher densities and the potentials for the strange quarks $(s, \bar{s})$ are given by the DQPM.
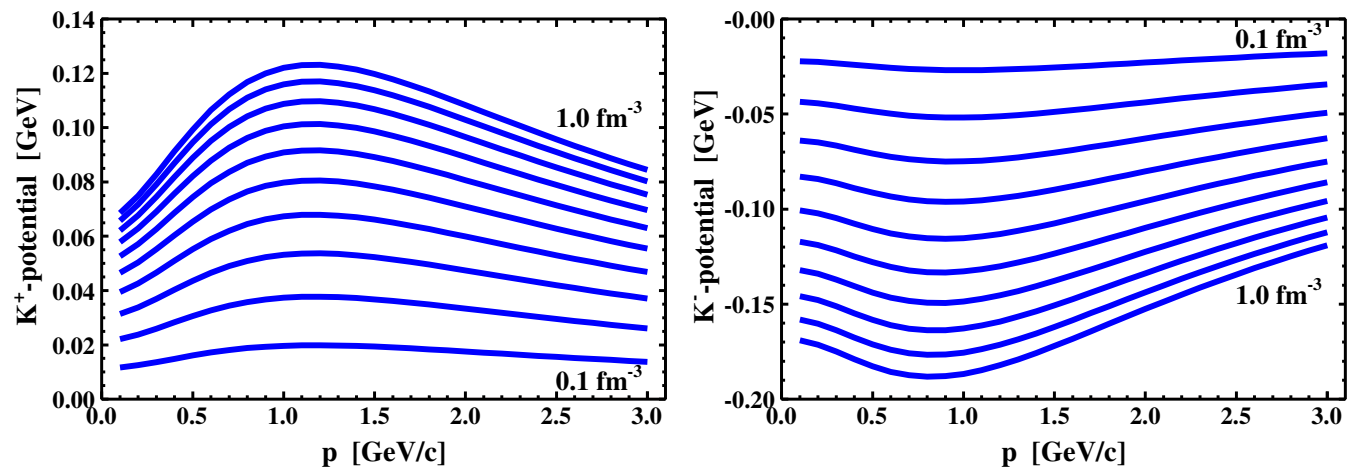

Figure 5. (1.h.s.) The repulsive kaon potential as a function of the momentum $p$ with respect to the local rest frame for nuclear densities from $0.1 \mathrm{fm}^{-3}$ to $1.0 \mathrm{fm}^{-3}$ in steps of $0.1 \mathrm{fm}^{-3}$. (r.h.s.) Same as on the 1.h.s. for the attractive antikaon potential employed.

\subsection{Predictions for directed kaon and antikaon flows}

We directly step on with the preliminary results for $\mathrm{Au}+\mathrm{Au}$ collisions at $\mathrm{b}=7 \mathrm{fm}$ for invariant energies of $\sqrt{s_{N N}}$ from 3 to $200 \mathrm{GeV}$, i.e. from the low FAIR/NICA energies to the top RHIC energy. The 

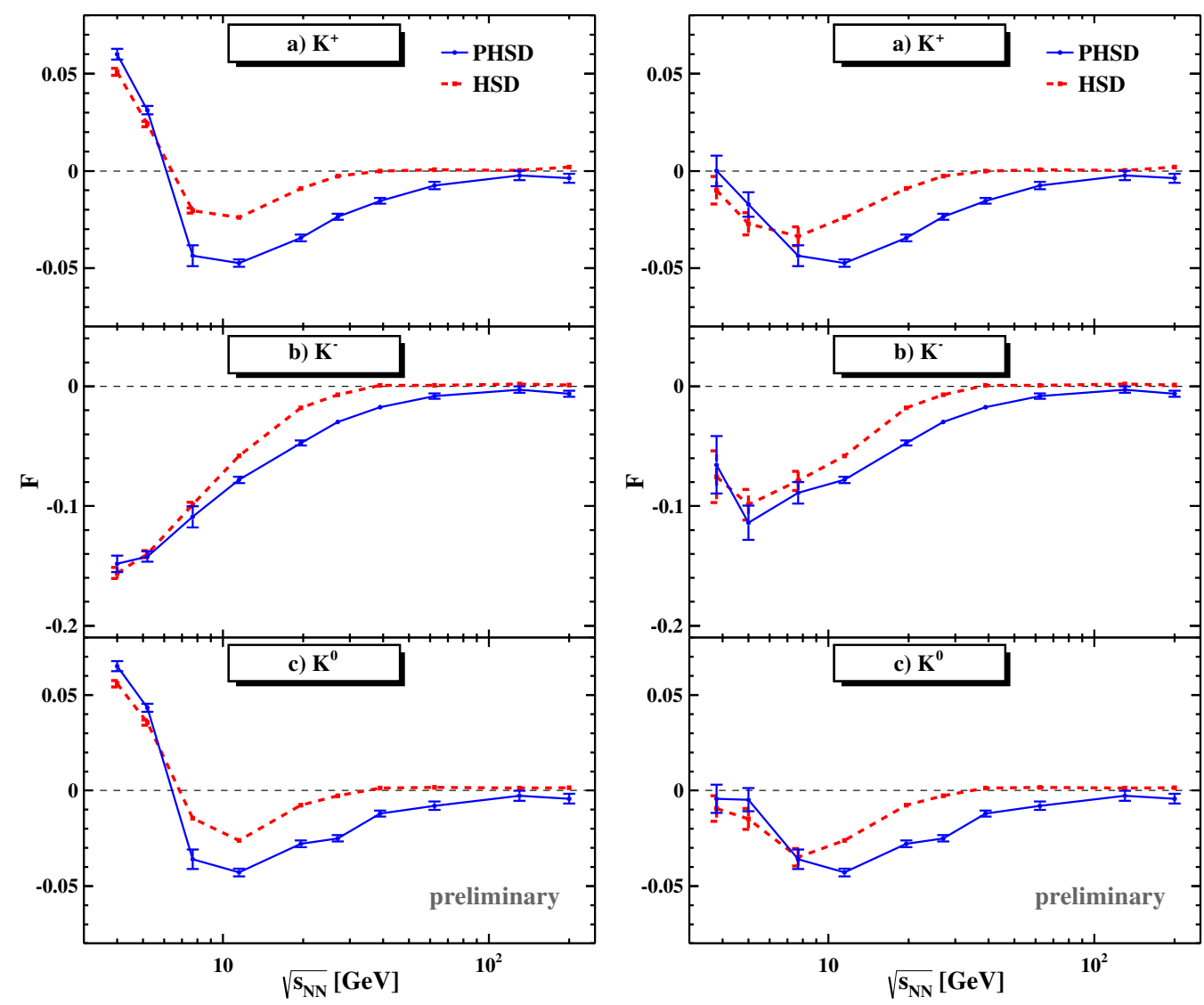

Figure 6. (1.h.s.) The directed flow slope $F$ for $K^{+}, K^{-}, K^{0}$ as a function of the invariant energy $\sqrt{s_{N N}}$ from lower FAIR/NICA to RHIC energies without kaon potentials. (r.h.s.) The directed flow slope $F$ for $K^{+}, K^{-}, K^{0}$ as a function of the invariant energy $\sqrt{s_{N N}}$ with kaon potentials in the HSD (dashed lines) and PHSD (solid lines) calculations.

actual results are shown in Fig. 6 from HSD (dashed lines) and PHSD (solid lines) for $K^{+}, K^{-}$and $K^{0}$ mesons, where the lefthand column corresponds to calculations without kaon potentials whereas the righthand column shows calculations with the kaon potentials from Fig. 5 included. As in Fig. 2 (r.h.s.) we find the same pattern (without potentials) as for pions above $\sqrt{s_{N N}} \approx 10 \mathrm{GeV}$. The PHSD calculations show a larger negative flow for kaons and antikaons than HSD; these directed flows are comparable in size with those from the pions. The latter is due to the fact that these hadrons dominantly emerge from parton fusion in the hadronization process. On the other hand, the HSD and PHSD give very similar results for $\sqrt{s_{N N}}<7 \mathrm{GeV}$ which has to be attributed to the dominance of hadronic degrees of freedom. Furthermore, we find a remarkable sensitivity to the kaon/antikaon potentials in this low energy (FAIR/NICA) domain when comparing the results from the left and right columns in Fig. 6. The positive flow of kaons even changes sign when including the repulsive potentials whereas the antikaon flow is decreased in size substantially by the attractive mean fields. Above about $\sqrt{s_{N N}}>10 \mathrm{GeV}$ there is no longer a sizeable sensitivity to the kaon/antikaon potentials. Accordingly, heavy-ion collisions at FAIR/NICA energies have the potential to provide information 
on the hadron properties (or dispersion relations) at high baryon densities while still at moderate temperatures.

\section{Conclusions}

In this study the parton-hadron-string dynamics (PHSD) approach has been applied for the analysis of the recent STAR data on the directed flow of identified hadrons [27] in the energy range $\sqrt{s_{N N}}=7.7$ $200 \mathrm{GeV}$. The excitation functions for the directed flows of protons, antiprotons and charged pions turn out to be smooth functions in bombarding energy without 'wiggle-like' irregularities [29] as expected before in Refs. [20-26]. Our results differ from the standard UrQMD model at lower bombarding energies as included in Ref. [27] and the recent theoretical analysis in Ref. [28]. The microscopic PHSD transport approach reproduces the general trend in the differential $v_{1}(y)$ excitation function and leads to an almost quantitative agreement for protons, antiprotons and pions especially at higher energies. We attribute this success to the Kadanoff-Baym dynamics incorporated in PHSD (with more accurate spectral functions) as compared to a Boltzmann-like on-shell transport model (UrQMD) and the account for parton dynamics also in this 'moderate' energy range. The latter is implemented in PHSD in line with the equation of state from lattice QCD [52]. The formation of the parton-hadron mixed phase softens the effective EoS in PHSD and describes a crossover transition (in line with the lattice QCD EoS). Accordingly, the PHSD results differ from those of HSD where no partonic degrees of freedom are incorporated. A comparison of both microscopic models has provided detailed information on the effect of parton dynamics on the directed flow (cf. Fig. 2).

Antiprotons have been shown to be particularly interesting. In HSD/PHSD we include antiproton annihilation into several mesons while taking into account also the inverse processes of $p \bar{p}$ creation in multi-meson interactions by detailed balance [45]. Related kinetic models (including UrQMD) which neglect the inverse processes for antiproton annihilation at lower energies do not describe the data on the directed flow of hadrons $v_{1}(y)$. We note in passing that 3FD hydrodynamics provides the best results with a crossover EoS for the quark-hadron phase transition [29] which by default is implemented in PHSD.

Still sizeable discrepancies with experimental measurements in the directed flow characteristics are found for the microscopic kinetic models at $\sqrt{s_{N N}} \leq 15 \mathrm{GeV}$ and are common for both HSD and PHSD (and UrQMD [39]) since the partonic degrees of freedom are subleading at these energies. We recall that the flow observables are not the only ones where the kinetic approaches have a problem in this energy range. Another long-standing issue is the overestimation of pion production as seen in Fig. 1 in the energy regime around the 'horn' in the $K^{+} / \pi^{+}$meson ratio [38, 59] which before has been related to a first-order phase transition or to the onset of deconfinement [60]. Our flow analysis shows no indication of a first-order transition in the energy range investigated. However, we have found further strong evidence that the dynamics of heavy-ion reactions at lower SPS or FAIR/NICA energies is far from being understood especially on the hadronic level without including hadronic mean fields (potentials).

On the other hand, we could demonstrate that kaon and antikaon potentials have a large impact on the kaon and antikaon flows for $\sqrt{s_{N N}} \leq 7 \mathrm{GeV}$ where the hadronic dynamics dominate. Presently, these potentials are not well known at high baryon densities and large momenta which provides the experimental perspective to shed further light on this issue. Furthermore, also baryon and antibaryon potentials will have an impact on the hadronic flow patterns as demonstrated in Refs. [5-7]. Note that the latter mean fields have been discarded in our present studies. We speculate that extended theoretical approaches including consistently chiral partners as well as a restoration of chiral symmetry at high baryon density and/or temperature might lead to a solution of the current problems as well as precise experimental studies at FAIR, NICA or within the BES II program at RHIC [8]. 


\section{Acknowledgments}

The authors are thankful to Yu. B. Ivanov for illuminating discussions and valuable suggestions as well as for related calculations within the 3FD approach.

\section{References}

[1] S. A. Voloshin, A. M. Poskanzer and R. Snellings, in Landolt-Boernstein New Series, I/23, p. 5-54, edited by R. Stock (Springer-Verlag, 2010).

[2] P. Sorensen, In Quark-Gluon Plasma 4, ed. by R. Hwa and X.N. Wang, World Scientific (2010).

[3] A.M. Poskanzer and S.A. Voloshin, Phys. Rev. C 58, 1671 (1998).

[4] STAR Collaboration, arXiv:1007.2613.

[5] P. Danielewicz, Nucl. Phys. A 673, 375 (2000).

[6] P. K. Sahu and W. Cassing, Nucl. Phys. A 712, 357 (2002).

[7] M. Isse, A. Ohnishi et al., Phys. Rev. C 72, 064908 (2005).

[8] P. Senger et al., Lect. Notes Phys. 814, 681 (2011).

[9] H. Sorge, Phys. Rev. Lett. 78, 2309 (1997).

[10] N. Herrmann, J. P. Wessels, and T. Wienold, Ann. Rev. Nucl. Part. Sci. 49, 581 (1999).

[11] E. Schnedermann and U. Heinz, Phys. Rev. Lett. 69, 2908 (1992).

[12] D. E. Kahana, D. Keane, Y. Pang, T. Schlagel and S. Wang, Phys. Rev. Lett. 74, 4404 (1995).

[13] J. Barrette et al. (E877 Collaboration), Phys. Rev. Lett. 73, 2532 (1994).

[14] I. G. Bearden et al. (NA44 Collaboration), Phys. Rev. Lett. 78, 2080 (1997).

[15] C. Alt et al. (NA49 Collaboration), Phys. Rev. C 68, 034903 (2003).

[16] E877 Collaboration, J. Barrette et al., Phys. Rev. C 55, 1420 (1997); J. Barrette et al., Phys. Rev. C 56, 3254 (1997).

[17] W. Reisdorf and H.G. Ritter, Annu. Rev. Nucl. Part. Sci. 47, 663 (1997).

[18] WA98 Collaboration, M.M. Aggarwal et al., nucl-ex/9807004.

[19] Yu. B. Ivanov et al., Acta Phys. Hung. New Ser. Heavy Ion Phys. 15, 117 (2002).

[20] D. H. Rischke et al., Heavy Ion Phys. 1, 309 (1995).

[21] D. H. Rischke, Nuclear Physics A 610, 88 (1996).

[22] C. M. Hung and E. V. Shuryak, Phys. Rev. Lett. 75, 4003 (1995).

[23] A.A. Shanenko and V.D. Toneev, JINR Rap. Com.5[73], 21 (1995); E.G. Nikonov, A.A. Shanenko and V.D. Toneev, Heavy Ion Phys. 4, 333 (1996).

[24] L. Mornas and U. Ornik. Nucl. Phys. A 587, 828 (1995).

[25] D. Rischke and M. Guylassy. Nucl. Phys. A 597, 4 (1996).

[26] H. Stöcker, Nucl. Phys. A 750, 121 (2005).

[27] STAR Collaboration: L. Adamczyk, et al., Phys. Rev. Lett. 112, 162301 (2014).

[28] J. Steinheimer et al., Phys. Rev. C 89, 054913 (2014).

[29] V. Konchakovski, W. Cassing and V. D. Toneev, Phys. Rev. C 90, 014903 (2014)

[30] S. Juchem, W. Cassing, and C. Greiner, Phys. Rev. D 69, 025006 (2004); Nucl. Phys. A 743, 92 (2004).

[31] W. Cassing, E. L. Bratkovskaya, Nucl. Phys. A 831, 215 (2009); Phys. Rev. C 78, 034919 (2008); W. Cassing, Nucl. Phys. A 791, 365 (2007).

[32] W. Cassing, E. Phys. J. ST 168, 3 (2009).

[33] V. P. Konchakovski et al., Phys. Rev. C 85, 011902 (2012). 
[34] O. Linnyk et al., Phys. Rev. C 84 (2011) 054917; Phys. Rev. C 85 (2012) 024910; Phys. Rev. C 87 (2013) 014905.

[35] V. D. Toneev et al., Phys. Rev. C 85, 034910 (2012).

[36] W. Ehehalt and W. Cassing, Nucl. Phys. A 602, 449 (1996).

[37] W. Cassing and E. L. Bratkovskaya, Phys. Rep. 308, 65 (1999).

[38] W. Cassing, E. L. Bratkovskaya, S. Juchem, Nucl. Phys. A 674, 249 (2000).

[39] E. L. Bratkovskaya et al., Phys. Rev. C 69, 054907 (2004).

[40] A. Andronic, P. Braun-Munzinger and J. Stachel, Nucl. Phys. A 772, 167 (2006).

[41] X. Zhu [STAR Collaboration], Acta Phys. Polon. Supp. 5, 213 (2012).

[42] C. Blume et al., https://edms.cern.ch/document/1075059

[43] C. Blume and C. Markert, Prog. Part. Nucl. Phys. 66, 834 (2011).

[44] H. Pi, Comp. Phys. Commun. 71, 173 (1992); T. Sjöstrand et al., Comp. Phys. Commun. 135, 238 (2001).

[45] W. Cassing, Nucl. Phys. A 700, 618 (2002).

[46] S.A. Bass et al., Prog. Part. Nucl. Phys. 42, 279 (1998).

[47] L.V. Bravina et al., J. Phys. G 25, 351 (1999); Phys. Rev. C 62, 064906 (2000).

[48] A.B. Larionov, W. Cassing, S. Leopold, and U. Mosel, Nucl. Phys. A 696, 619 (2001).

[49] A. Jahns, C. Spieles, H. Sorge, Horst Stöcker, and W. Greiner, Phys. Rev. Lett. 72, 3464 (1994).

[50] H. Liu et al. (E895 Collaboration), Phys. Rev. Lett. 84, 5488 (2000).

[51] J. Barrette et al. (E877 Collaboration), Phys. Lett. B 485, 319 (2000).

[52] Y. Aoki et al., Phys. Lett. B 643, 46 (2006); S. Borsanyi et al., JHEP 1009, 073 (2010); JHEP 1011, 077 (2010); JHEP 1208, 126 (2012); Phys. Lett. B 730, 99 (2014).

[53] G.-Q. Li, C. M. Ko, and X. S. Fang, Phys. Lett. B 329, 149 (1994).

[54] J. Schaffner, A. Gal, I. N. Mishustin, H. Stöcker, and W. Greiner, Phys. Lett. B 334, 268 (1994).

[55] E.L. Bratkovskaya, W. Cassing, and U. Mosel, Nucl. Phys. A 622, 593 (1997).

[56] H. Oeschler, Y. Leifels, E. L. Bratkovskaya, and J. Aichelin, Phys. Rept. 510, 119 (2012).

[57] L. Tolós, A. Ramos, and A. Polls, Phys. Rev. C 65, 054907 (2002).

[58] W. Cassing, L. Tolós, E. L. Bratkovskaya, and A. Ramos, Nucl. Phys. A 727, 59 (2003).

[59] M. Gazdzicki and M. I. Gorenstein, Acta Phys. Polon. B 30, 2705 (1999).

[60] M. Gazdzicki, M. Gorenstein and P. Seyboth, Acta Phys. Polon. B 42, 307 (2011). 